\title{
Universiteit
}

Leiden

The Netherlands

\section{Ejection and Capture Dynamics in Restricted Three-body Encounters}

Kobayashi, S.; Hainick, Y.; Sari, R.; Rossi, E.M.

\section{Citation}

Kobayashi, S., Hainick, Y., Sari, R., \& Rossi, E. M. (2012). Ejection and Capture Dynamics in Restricted Three-body Encounters. The Astrophysical Journal, 748(2), 105.

doi:10.1088/0004-637X/748/2/105

Version: $\quad$ Not Applicable (or Unknown)

License: $\quad$ Leiden University Non-exclusive license

Downloaded from: https://hdl.handle.net/1887/45918

Note: To cite this publication please use the final published version (if applicable). 


\title{
EJECTION AND CAPTURE DYNAMICS IN RESTRICTED THREE-BODY ENCOUNTERS
}

\author{
Shiho Kobayashi ${ }^{1}$, Yanir Hainick ${ }^{2,3}$, Re'em SARI $^{3,4}$, and Elena M. Rossi ${ }^{5}$ \\ ${ }^{1}$ Astrophysics Research Institute, Liverpool John Moores University, Birkenhead CH41 1LD, UK \\ ${ }^{2}$ Raymond and Beverly Sackler School of Physics and Astronomy, Tel Aviv University, Tel Aviv 69978, Israel \\ ${ }^{3}$ Racah Institute of Physics, Hebrew University, Jerusalem 91904, Israel \\ 4 Theoretical Astrophysics 350-17, California Institute of Technology, CA 91125, USA \\ ${ }^{5}$ Leiden Observatory, Leiden University, P.O. Box 9513, 2300 RA Leiden, The Netherlands \\ Received 2011 November 22; accepted 2012 January 20; published 2012 March 13
}

\begin{abstract}
We study the tidal disruption of binaries by a massive point mass (e.g., the black hole at the Galactic center), and we discuss how the ejection and capture preference between unequal-mass binary members depends on which orbit they approach the massive object. We show that the restricted three-body approximation provides a simple and clear description of the dynamics. The orbit of a binary with mass $m$ around a massive object $M$ should be almost parabolic with an eccentricity of $|1-e| \lesssim(m / M)^{1 / 3} \ll 1$ for a member to be captured, while the other is ejected. Indeed, the energy change of the members obtained for a parabolic orbit can be used to describe non-parabolic cases. If a binary has an encounter velocity much larger than $(M / m)^{1 / 3}$ times the binary rotation velocity, it would be abruptly disrupted, and the energy change at the encounter can be evaluated in a simple disruption model. We evaluate the probability distributions for the ejection and capture of circular binary members and for the final energies. In principle, for any hyperbolic (elliptic) orbit, the heavier member has more chance to be ejected (captured), because it carries a larger fraction of the orbital energy. However, if the orbital energy is close to zero, the difference between the two members becomes small, and there is practically no ejection and capture preferences. The preference becomes significant when the orbital energy is comparable to the typical energy change at the encounter. We discuss its implications to hypervelocity stars and irregular satellites around giant planets.
\end{abstract}

Key words: binaries: general - Galaxy: center - Galaxy: halo - Galaxy: kinematics and dynamics - planets and satellites: formation - planets and satellites: individual (Triton)

Online-only material: color figures

\section{INTRODUCTION}

The disruption of a star by a massive black hole (BH) is one of the most spectacular examples of the tidal phenomena (Komossa \& Bade 1999; Donley et al. 2002; Grupe et al. 1995). A star that wanders too close to a massive $\mathrm{BH}$ is torn apart by gravitational forces. Almost half the debris would escape on hyperbolic orbits, while the other half would traverse elliptic orbits and return to periapsis before producing a conspicuous flare (e.g., Rees 1988). The disruption process has been numerically investigated in detail (Evans \& Kochanek 1989; Laguna et al. 1993; Ayal et al. 2000; Kobayashi et al. 2004; Guillochon et al. 2009), and the new generation of all-sky surveys are expected to detect many tidal flares (Strubbe \& Quataert 2009; Lodato \& Rossi 2011). Recently, a possible discovery of the onset of rapid $\mathrm{BH}$ accretion has been reported (Burrows et al. 2011; Zauderer et al. 2011; Levan et al. 2011; Bloom et al. 2011).

Once a star gets deeply inside the tidal radius of a $\mathrm{BH}$, the tidal force dominates over the self-gravity and thermal pressure of the star. A very simplified description of the disruption process could be the encounter between a star cluster (or a cluster of point masses) and a massive $\mathrm{BH}$. The simplest case consists of a binary and a massive $\mathrm{BH}$ in which after the tidal disruption, one star would escape to infinity, while the other could be captured by the $\mathrm{BH}$. This is actually one of the leading models for the formation of hypervelocity stars (Hills 1988; Yu \& Tremaine 2003). The captured stars may explain the S-stars in the Galactic center (Gould \& Quillen 2003; Ginsburg \& Loeb 2006; Ghez et al. 2005; Genzel et al. 2010).

Hypervelocity stars are stars with a high velocity exceeding the escape velocity of the Galaxy. After the discovery of such stars in a survey of blue stars within the Galactic halo (Brown et al. 2005; Hirsch et al. 2005; Edelmann et al. 2005), many authors have predicted the properties of hypervelocity stars (Gualandris et al. 2005; Bromley et al. 2006; Sesana et al. 2007; Perets et al. 2007; Kenyon et al. 2008; Tutukov \& Fedorova 2009; Antonini et al. 2011; Zhang et al. 2010). These investigations so far have used three-body simulations or analytic methods that relied on results from three-body simulations.

The six orders of magnitude mass ratio between the Galactic center BH and the binary stars allows us to formulate the problem in the restricted three-body approximation. In a previous paper (Sari et al. 2010, hereafter SKR), we have shown that the approximation is efficient and useful to understand how binary stars behave at the tidal breakup when the binary's center of mass approaches the $\mathrm{BH}$ in a parabolic orbit. In this paper, we generalize the approximation for orbits with arbitrary eccentricity. This enables us to give a complete picture of the ejection and capture process. We also provide the ejection and capture probability distributions that can be simply rescaled in terms of binary masses, their initial separation, and the binary-to-black hole mass ratio when applied to a specific system. Our method is computationally more efficient than full three-body simulations, and it is easier to grasp the nature of the tidal interaction.

In Section 2, we outline the restricted three-body approximation. In Section 3, we evaluate how much energy each member gains or loses at the tidal encounter and we discuss how the energy change evaluated for a parabolic orbit can be used to study non-parabolic orbit cases, and in Section 4, we give qualitative discussion on the ejection and capture preferences. In Section 5, we study high-velocity encounters. In Section 6, the numerical 
results are discussed. In Section 7, we use our results to describe the capture process of Triton around Neptune. Finally, in Section 8 , we summarize the results.

\section{THE RESTRICTED THREE-BODY PROBLEM}

The equation of motion for each of the binary members is given by

$$
\begin{aligned}
& \ddot{\mathbf{r}}_{1}=-\frac{G M}{r_{1}^{3}} \mathbf{r}_{1}+\frac{G m_{2}}{\left|\mathbf{r}_{1}-\mathbf{r}_{2}\right|^{3}}\left(\mathbf{r}_{2}-\mathbf{r}_{1}\right), \\
& \ddot{\mathbf{r}}_{2}=-\frac{G M}{r_{2}^{3}} \mathbf{r}_{2}-\frac{G m_{1}}{\left|\mathbf{r}_{1}-\mathbf{r}_{2}\right|^{3}}\left(\mathbf{r}_{2}-\mathbf{r}_{1}\right),
\end{aligned}
$$

where $\mathbf{r}_{1}$ and $\mathbf{r}_{2}$ are the respective distance from the massive point mass with $M$. We will call the point mass the $\mathrm{BH}$, though the binary is assumed to travel well outside the event horizon and our results can be applied to any systems which include a Newtonian massive point mass. The equation for the distance between the two $\mathbf{r} \equiv \mathbf{r}_{2}-\mathbf{r}_{1}$ is

$$
\ddot{\mathbf{r}}=-\frac{G M}{r_{2}^{3}} \mathbf{r}_{2}+\frac{G M}{r_{1}^{3}} \mathbf{r}_{1}-\frac{G m}{r^{3}} \mathbf{r},
$$

where $m=m_{1}+m_{2} \ll M$. We assume that the two masses are much closer to each other, and to the trajectory of the center of mass of the binary $\mathbf{r}_{\mathrm{m}}$, than each of them to the BH. Both energy and orbit obtained under the approximation are fairly accurate except for a part of the orbit just around the periapsis passage (see SKR for the details).

Linearizing the first two terms of Equation (3) around the center of mass orbit $\mathbf{r}_{\mathrm{m}}$, we find that the zero orders cancel out. Then, rescaling the distance between the bodies by $(m / M)^{1 / 3} r_{\mathrm{p}}$ and the time by $\sqrt{r_{\mathrm{p}}^{3} / G M}$, where $r_{\mathrm{p}}$ is the distance of the closest approach between the center of mass of the binary and the $\mathrm{BH}$, we can re-write Equation (3) in terms of the dimensionless variables: $\boldsymbol{\eta} \equiv(M / m)^{1 / 3}\left(\mathbf{r} / r_{\mathrm{p}}\right)$ and $t$ :

$$
\ddot{\boldsymbol{\eta}}=\left(\frac{r_{\mathrm{p}}}{r_{\mathrm{m}}}\right)^{3}\left[-\boldsymbol{\eta}+3\left(\boldsymbol{\eta} \hat{\mathbf{r}}_{\mathrm{m}}\right) \hat{\mathbf{r}}_{\mathrm{m}}\right]-\frac{\boldsymbol{\eta}}{|\boldsymbol{\eta}|^{3}},
$$

where $\hat{\mathbf{r}}_{\mathrm{m}}$ is a unit vector pointing the center of mass of the binary. We define the orbit of the center of mass to be a conic orbit $r_{\mathrm{m}} / r_{\mathrm{p}}=(1+e) /(1+e \cos f)$, where $e$ is the eccentricity and the true anomaly $f$ is the angle from the point of closest approach. Since $\hat{\mathbf{r}}_{\mathrm{m}}=(\cos f, \sin f, 0)$, and we set $\eta=(x, y, z)$, explicit equations in terms of dimensionless Cartesian coordinates read

$$
\begin{aligned}
\ddot{x}= & \frac{(1+e \cos f)^{3}}{(1+e)^{3}}[-x+3(x \cos f+y \sin f) \cos f] \\
& -\frac{x}{\left(x^{2}+y^{2}+z^{2}\right)^{3 / 2}}, \\
\ddot{y}= & \frac{(1+e \cos f)^{3}}{(1+e)^{3}}[-y+3(x \cos f+y \sin f) \sin f] \\
& -\frac{y}{\left(x^{2}+y^{2}+z^{2}\right)^{3 / 2}}, \\
\ddot{z}= & -\frac{(1+e \cos f)^{3}}{(1+e)^{3}} z-\frac{z}{\left(x^{2}+y^{2}+z^{2}\right)^{3 / 2}},
\end{aligned}
$$

where the eccentricity

$$
e=1+\frac{2 r_{\mathrm{p}} E}{G M m}
$$

is related to the energy of the center of mass which is given by

$$
E=\frac{m}{2}\left|\dot{\mathbf{r}}_{\mathrm{m}}\right|^{2}-\frac{G M m}{r_{\mathrm{m}}} .
$$

Using the dimensionless time, the conservation of the angular momentum can be expressed as

$$
\dot{f}=(1+e)^{-3 / 2}(1+e \cos f)^{2} .
$$

Analytically, one has relations between $r_{\mathrm{m}}$ and $t$ through a parameter which are given by (e.g., Landau \& Lifshitz 1976)

$$
\begin{aligned}
E<0 \quad r_{\mathrm{m}} / r_{\mathrm{p}} & =(1-e)^{-1}(1-e \cos \xi), \\
t & =(1-e)^{-3 / 2}(\xi-e \sin \xi), \\
E=0 \quad r_{\mathrm{m}} / r_{\mathrm{p}} & =\left(1+\xi^{2}\right), \quad t=\sqrt{2}\left(\xi+\xi^{3} / 3\right), \\
E>0 \quad r_{\mathrm{m}} / r_{\mathrm{p}} & =(e-1)^{-1}(e \cosh \xi-1), \\
t & =(e-1)^{-3 / 2}(e \sinh \xi-\xi),
\end{aligned}
$$

where the closest approach $r_{\mathrm{m}}=r_{\mathrm{p}}$ happens at $t=0$.

\section{ENERGY CHANGE AT THE BH ENCOUNTER}

We are interested in the fate of stars in a binary, following its encounter with a massive $\mathrm{BH}$. In order to study the ejection and capture process, we evaluate the energies of the stars as functions of time. When the binary is at a large distance from the $\mathrm{BH}$, the binary members rotate around their center of mass which gradually accelerates toward the $\mathrm{BH}$. The specific selfgravity energy of the binary is about $-v_{0}^{2} \equiv-G m / a$. Analytic arguments (SKR) suggest that at the tidal breakup one member gets additional energy of the order of $v_{\mathrm{m}} v_{0}$, where $v_{\mathrm{m}}$ is the velocity of the center of mass at the tidal radius $r_{t}=(M / m)^{1 / 3} a$. If the binary approaches the $\mathrm{BH}$ with negligible orbital energy, the velocity is $v_{\mathrm{m}}=\left(G M / r_{t}\right)^{1 / 2}=v_{0}(M / m)^{1 / 3}$. The additional energy is larger than the self-gravity energy by a factor of $(M / m)^{1 / 3} \gg 1$. Therefore, we will neglect the self-gravity term in the following energy estimates. This treatment is valid as long as the binary is injected into the orbit $\mathbf{r}_{\mathrm{m}}$ at a radius much larger than the tidal radius.

The energy of one binary member $m_{i}$ is given by

$$
E_{i}=\frac{m_{i}}{2}\left|\dot{\mathbf{r}}_{i}\right|^{2}-\frac{G M m_{i}}{r_{i}} .
$$

Linearizing the kinetic and potential energy terms around the orbit of the center of mass $\mathbf{r}_{\mathrm{m}}$ and using the initial energy $I_{i} \equiv\left(m_{i} / m\right) E$, we obtain

$$
\begin{gathered}
E_{i}=I_{i}+\Delta E_{i} \\
\Delta E_{i} \equiv m_{i} \dot{\mathbf{r}}_{\mathrm{m}}\left(\dot{\mathbf{r}}_{i}-\dot{\mathbf{r}}_{\mathrm{m}}\right)+\frac{G M m_{i}}{r_{\mathrm{m}}^{3}} \mathbf{r}_{\mathrm{m}}\left(\mathbf{r}_{i}-\mathbf{r}_{\mathrm{m}}\right)
\end{gathered}
$$


Since in our limit the total energy of the system is $E$, considering $\Delta E_{2}=-\Delta E_{1}$, we get

$$
\Delta E_{2}=\frac{m_{1} m_{2}}{m}\left(\dot{\mathbf{r}}_{\mathrm{m}} \dot{\mathbf{r}}+\frac{G M}{r_{\mathrm{m}}^{3}} \mathbf{r}_{\mathrm{m}} \mathbf{r}\right) .
$$

Using our rescaled variables, the additional energy is given by

$$
\begin{aligned}
\Delta E_{2}= & -\Delta E_{1}=\frac{G m_{1} m_{2}}{a}\left(\frac{M}{m}\right)^{1 / 3} \Delta \bar{E}, \\
\Delta \bar{E} \equiv & D^{-1}\left[\frac{-\dot{x} \sin f+\dot{y}(e+\cos f)}{\sqrt{1+e}}\right. \\
& \left.+\frac{(1+e \cos f)^{2}}{(1+e)^{2}}(x \cos f+y \sin f)\right],
\end{aligned}
$$

where $D=r_{\mathrm{p}} / r_{t}$ is the penetration factor which is useful to characterize the tidal encounter. Once the binary dissolves, $\Delta \bar{E}$ becomes a constant because the body is eventually moving only under the conservative force of the $\mathrm{BH}$. Hereafter, the energy change $\Delta \bar{E}$ means the constant value after the disruption, otherwise we specify it. The equation of motion (4) indicates that the negative of a solution $\mathbf{r}=\mathbf{r}(t)$ is also a solution. The energies $\Delta E_{i}$ are also linear in the coordinates. Therefore, another binary starting with a phase difference $\pi$ will have the same additional energy in absolute value but opposite in sign. A uniform distribution in the binary phase implies that, when the binary is disrupted, each body has a $50 \%$ chance of gaining energy (and a 50\% chance of losing energy).

As we have discussed, the typical energy change is larger than the self-gravity energy by a factor of $\sim(M / m)^{1 / 3}$, it is of the order of $\left(G m_{1} m_{2} / a\right)(M / m)^{1 / 3}$. Then, the dimensionless quantity $\Delta \bar{E}$ is an order-of-unity constant after the disruption. Its exact value depends on orbital parameters, but for qualitative discussion we just need to know that $\Delta \bar{E}$ is about unity. Later, we will numerically show that $\Delta \bar{E}$ is an order of unity in the relevant parameter regime, ${ }^{6}$ and numerical values will be used to estimate the ejection and capture probabilities.

Rescaling energies by the typical value of the energy change, the energies of the binary members after the disruption are given by

$$
\bar{E}_{1}=\bar{I}_{1}-\Delta \bar{E}, \quad \bar{E}_{2}=\bar{I}_{2}+\Delta \bar{E},
$$

where bar denotes energy scaled by $\left(G m_{1} m_{2} / a\right)(M / m)^{1 / 3}$. An interesting outcome of the encounter between a binary system and a massive $\mathrm{BH}$ is the "three-body exchange reaction" (Heggie 1975; Hills 1975) where one member of the binary is expelled and its place is taken by the $\mathrm{BH}$, i.e., one binary member is captured by the $\mathrm{BH}$ and the other is ejected to infinity. In order for a member $m_{i}$ to escape from the $\mathrm{BH}$, the initial binding energy should be smaller than the energy gain: $\left|\bar{I}_{i}\right|<|\Delta \bar{E}| \sim 1$. The same condition is required when a member of the binary in a hyperbolic orbit loses energy and is bound around the $\mathrm{BH}$. Therefore, when we discuss the ejection or capture process associated with a massive $\mathrm{BH}$, the absolute value of the initial energy should be comparable or less than unit: $\left|\bar{I}_{i}\right| \lesssim 1$.

\footnotetext{
6 For prograde orbits with $D \sim 0.1$, the energy change is as large as $\Delta \bar{E} \sim 30$ in a very narrow range of the binary phase (see Figure 7 in SKR) where the binary members once come close to each other before they break up. However, the phase-averaged value $\langle|\Delta \bar{E}|\rangle$ is still an order of unity and it is a more relevant quantity for the discussion on the ejection and capture probabilities and the final energies.
}

Since the energy, penetration factor (periapsis radius), and eccentricity are related by Equation (8) or equivalently

$$
e=1+2 D \bar{E}\left(\frac{m}{M}\right)^{1 / 3}\left(\frac{m_{1}}{m}\right)\left(\frac{m_{2}}{m}\right),
$$

only two of them are independent parameters used to describe the binary orbit. Considering $\left|\bar{I}_{i}\right| \lesssim 1$ together with the mass ratio $(m / M)^{1 / 3} \ll 1$ and the tidal disruption condition $D \lesssim 1$, the eccentricity should be almost unity $|1-e| \lesssim$ $D(\tilde{m} / M)^{1 / 3}\left(m_{\text {par }} / m\right)$ for a member $m_{i}$ to be ejected or captured where $m_{\mathrm{par}}$ is the mass of the partner $\left(m_{\mathrm{par}}=m_{2}\right.$ for $i=1$ and $m_{1}$ for $\left.i=2\right)$. If we use the semimajor axis $r_{a} \equiv r_{t} D /(1-e)$, the condition can be rewritten as $\left|r_{a} / r_{t}\right| \gtrsim(M / m)^{1 / 3}\left(m / m_{\text {par }}\right)$ where $r_{a}$ is negative for hyperbolic orbits.

Such orbits differ very little from parabolic orbits with the same periapsis distance, especially around the tidal radius and inside it. Therefore, the energy change $\Delta \bar{E}$ is expected to be almost identical to that for the parabolic case. As long as we study the exchange reaction, we can approximate $\Delta \vec{E}$ by the parabolic results $\Delta \bar{E}_{e=1}$. However, $e \sim 1$ does not necessarily mean $|\bar{E}| \ll 1$. In general, we need to take into account the offset of the final energy due to the non-zero initial energy, which would affect the ejection and capture probabilities. The final energies are approximately given by

$$
\bar{E}_{1}=\bar{I}_{1}-\Delta \bar{E}_{e=1}, \quad \bar{E}_{2}=\bar{I}_{2}+\Delta \bar{E}_{e=1} .
$$

\section{WHICH GETS KICKED OUT?}

We here consider a simple question: which member is ejected or captured if an unequal-mass binary is tidally disrupted by a massive $\mathrm{BH}$ ? If $\bar{E}>0$ (hyperbolic orbits), then one binary member could lose energy and get captured by the $\mathrm{BH}$, while the other flies away with a larger energy. Assuming a uniform distribution in the binary phase, each member has a $50 \%$ chance of losing energy (and gaining energy). However, since the lighter one (the secondary) has a smaller initial energy, it is preferentially captured and the heavier one (the primary) has more chance to be ejected.

For elliptical orbits, by considering a plausible semimajor axis $r_{a}$, we can obtain tighter constraints on the eccentricity and energy, compared to the requirements from the exchange reaction. This is particularly relevant for studies of hypervelocity stars. If $r_{a}$ is around the radius of influence of the $\mathrm{BH} r_{\mathrm{h}} \sim$ $G M / \sigma^{2}$ where $\sigma$ is the local stellar velocity dispersion, for the Galactic center, it is about $r_{a} \sim$ a few parsecs $\sim 10^{5} r_{t}$ for $a \sim$ several solar radii. Then, we get $1-e=D\left(r_{t} / r_{a}\right) \lesssim 10^{-5}$ and $|\bar{E}| \sim\left(r_{t} / r_{a}\right)(M / m)^{1 / 3}\left(m / m_{1}\right)\left(m / m_{2}\right) \sim 10^{-3}$. Our previous estimates based on parabolic orbits are appropriate to study the production of hypervelocity stars for which an equal ejection chance is expected (SKR). When the semimajor axis is as small as $r_{a} \sim(M / m)^{1 / 3}\left(m / m_{\text {par }}\right) r_{t}$, the initial energy $\left|\bar{I}_{i}\right|$ would be of the order of unity, as we have discussed, and affect the ejection preference. Since the secondary has less negative initial energy, it is preferentially ejected.

Recently, Antonini et al. (2011) performed $N$-body simulations of unequal-mass binaries with $m_{1}=6 M_{\odot}, m_{2}=1$ or $3 M_{\odot}$, and $a=0.1 \mathrm{AU}$ in elliptical orbits around a supermassive $\mathrm{BH} M=4 \times 10^{6} M_{\odot}$. They find that the initial distance of the binary from the central BH plays a fundamental role in determining which member is ejected: for a large initial distance $d=0.1 \mathrm{pc}$, or equivalently $r_{a} \sim 3 \times 10^{3} r_{t}$, the ejection probability is almost independent of the stellar 
mass, while for $d=0.01 \mathrm{pc}$ or $r_{a} \sim 3 \times 10^{2} r_{t}$, the lighter star is preferentially ejected. Considering that the ejection probability significantly decreases if $r_{a}$ becomes smaller than $\sim(M / m)^{1 / 3}\left(m / m_{\text {par }}\right) r_{t} \sim 80\left(m / m_{\text {par }}\right) r_{t}$, these results are consistent with our analysis.

These ejection preferences for hyperbolic and elliptic orbits are naturally understood if we consider a large mass ratio for the binary members. The energy of the primary practically does not change at the tidal encounter. Whether it is ejected or captured after the tidal breakup simply depends on the initial energy $\sim E$, while the secondary might have a chance to make a transition between bound and unbound orbits around the BH (Bromley et al. 2006). In the large mass ratio limit, the exchange reaction condition (i.e., the transition condition for the secondary) is $|E| \lesssim\left(G m^{2} / a\right)(M / m)^{1 / 3}$ or equivalently $\left|r_{a}\right| \gtrsim(M / m)^{2 / 3} a$.

\section{HIGH-ENERGY REGIME}

If a binary has a large orbital energy $\bar{E} \gg 1$, then both members are ejected after the $\mathrm{BH}$ encounter as a binary system or two independent objects. ${ }^{7}$ Although the high-energy regime is not important in the context of the three-body exchange reaction, we discuss the regime to clarify the parameter dependence of the numerical results in the next section. A high orbital energy $\bar{E} \gg(M / m)^{1 / 3}\left(m / m_{1}\right)\left(m / m_{2}\right)$ affects the velocity of their center of mass at the encounter $v_{m} \sim\left(E / m+G M / r_{\mathrm{m}}\right)^{1 / 2}=$ $(M / m)^{1 / 3} v_{0} \sqrt{(e-1) / 2 D+r_{t} / r_{\mathrm{m}}}$. Then, the tidal disruption radius (i.e., where a binary is disrupted) can be defined in three different ways. We here order them from a large to small radius. (1) Relative acceleration: the radius at which the $\mathrm{BH}$ tidal force becomes comparable to the mutual gravity of the binary. This is $r_{t}$. (2) Relative velocity: the radius at which the tidal force induces the relative velocity between the binary members comparable to the binary escape velocity $v_{0}$. (3) Relative position: the radius at which the difference in position increases by more than the initial binary separation.

The duration that the center of mass is around $r_{\mathrm{m}}$ is of the order of $\Delta t \sim r_{\mathrm{m}} / v_{m}$. During this period, the tidal acceleration of the relative motion of the binary members by the $\mathrm{BH}$ is of the order of $A \sim G M a / r_{m}^{3}$. The two radii (2) and (3) can be estimated from two conditions: $\Delta v=A \Delta t \sim v_{0}$ and $\Delta x=A \Delta t^{2} \sim a$, provided that the duration of the encounter is comparable to or shorter than the binary rotation timescale: $\Delta t \lesssim a / v_{0}$. If the energy is high $(e-1) / D=2 \bar{E}(m / M)^{1 / 3}\left(m_{1} / m\right)\left(m_{2} / m\right) \gg 1$, then these conditions give $r_{\mathrm{m}}=r_{t} D^{1 / 4} /(e-1)^{1 / 4}$ and $r_{t} D /(e-1)$, respectively. Since they should be larger than the periapsis distance, only the cases that satisfy $D \lesssim(e-1)^{-1 / 3}$ for the radius (2) or $e \lesssim 2$ for the radius (3) lead to the disruption. The radius (3) is basically the place at which the orbit of the center of mass makes its turn (i.e., the periapsis). If the energy is low $(e-1) / D \ll 1$, then all the estimates give the original tidal radius $r_{t}$.

When we discuss the energy change $\Delta E$ at the tidal encounter, there are two important points which we should emphasize. First, the energy of each of the binary members in the $\mathrm{BH}$ frame changes only due to the mutual force between the binary members. Second, most of the work done by one member on the other, which is $\Delta E$, is done outside the tidal radius $r_{t}$. The mutual force is of the order of $G m_{1} m_{2} / a^{2}$. During the binary rotation timescale $a / v_{0}$, the force acts over a length $\sim\left(v_{m} / v_{0}\right) a$ in the $\mathrm{BH}$

\footnotetext{
7 If $\bar{E}$ is a large negative value, then both members are captured after the disruption. Since the velocity at the tidal radius is reduced $v_{m} \lesssim(M / m)^{1 / 3} v_{0}$, the energy change should be smaller $|\Delta \bar{E}| \lesssim 1$.
}

frame. Therefore, the work is $W \sim\left(m_{1} m_{2} / m\right) v_{m} v_{0}$. Since the direction of the mutual force changes with the binary rotation, $\Delta E(t)$ oscillates with the amplitude of $W$. When the binary is disrupted, $\Delta E$ becomes a constant value which is basically determined by the binary phase at the disruption. Then, we might expect that the final value of $\Delta E$ is a sinusoidal function of the binary phase for circular binaries. As we will see later, this is actually the case for the high-energy encounters. Even with the largest estimate of the tidal radius (i.e., $r_{t}$ ), the duration of the encounter $\Delta t \sim r_{t} / v_{m}$ is shorter than the binary rotation timescale by a factor of $\sim \sqrt{D /(e-1)} \ll 1$. The work during the encounter is negligible compared to the work $W$ which has been done outside $r_{t}$. On the other hand, in the low-energy regime, the duration of the encounter is comparable to the binary rotation timescale. Considering that at the encounter the orbits of the members in the comoving frame of their center of mass should be significantly deformed from the original orbits (e.g., circular orbits) before they finally break up, the work during the encounter could induce deviation of $\Delta E(\phi)$ from a simple sinusoidal function. However, the typical value is still expected to be about $\Delta E \sim\left(m_{1} m_{2} / m\right) v_{0} v_{m}$.

In both the low- and high-energy regime, the typical energy change is given in a dimensionless form by

$$
\begin{aligned}
\Delta \bar{E} & \sim \sqrt{1+\bar{E}\left(\frac{m}{M}\right)^{1 / 3}\left(\frac{m_{1}}{m}\right)\left(\frac{m_{2}}{m}\right)} \\
& =\sqrt{1+\frac{e-1}{2 D}}=\sqrt{1-\frac{r_{t}}{2 r_{a}}},
\end{aligned}
$$

where we have assumed $r_{\mathrm{m}}=r_{t}$ to estimate $v_{m}$. In the highenergy regime, the disruption might happen at a smaller radius, but $v_{m}$ is determined by the orbital energy and it is insensitive to the choice of $r_{\mathrm{m}}$. When $(e-1) / D \gg 1$, the energy change becomes much larger than unity. However, the energy gain is not significant compared to the original energy $\bar{E}$, and one finds that the tidal encounter is not an efficient acceleration process anymore.

In the high-energy regime, the energy change Equation (19) can be evaluated by assuming that a binary is abruptly disrupted at the tidal radius $r_{t}$, since the work during the tidal encounter is negligible. For a circular coplanar binary: $(x, y)=$ $D^{-1}\left(\cos \phi_{t}, \sin \phi_{t}\right)$ and $(\dot{x}, \dot{y})= \pm D^{1 / 2}\left(-\sin \phi_{t}, \cos \phi_{t}\right)$, we obtain

$$
\begin{aligned}
\Delta \bar{E}= & \left(1 \pm \frac{1}{\sqrt{D(1+e)}}\right) \sin f_{t} \sin \phi_{t} \\
& +\left(1 \pm \frac{1+e / \cos f_{t}}{\sqrt{D(1+e)}}\right) \cos f_{t} \cos \phi_{t}
\end{aligned}
$$

where $\phi_{t}$ is the binary phase at the tidal radius, $f_{t}$ is the negative value solution of $1+e \cos f_{t}=(1+e) D$, and the signature indicates a prograde $(+)$ or retrograde $(-)$ orbit. This is a sinusoidal function of the binary phase as we expected, and the square of its amplitude is $3-r_{t} / r_{a} \pm 2 \sqrt{(1+e) D}$, which is larger for prograde orbits and the difference between prograde and retrograde orbits becomes smaller for deep penetrators $D \ll 1$, because in this limit the binary center of mass approaches the $\mathrm{BH}$ in an almost radial fashion.

If the disruption is abrupt, then the ejection and capture preference could be roughly illustrated in terms of velocity (e.g., Morbidelli 2006; Agnor \& Hamilton 2006). The binary members rotate around their center of mass, such that their own motion is half of the time with and half of the time against, 
the motion of the center of mass $\dot{\mathbf{r}}_{\mathrm{m}}$. The net velocity of the members relative to the $\mathrm{BH}$ is accordingly increased or reduced. Since the secondary has a higher rotation velocity, it has more of a chance that the net velocity exceeds or drops below the escape velocity from the BH. Then, it is preferentially ejected to infinity or captured in a bound orbit. However, for the full discussion of the process, we also need to take into account the variation in the escape velocity or the variation in the potential. The displacement of order $a$ in the position of each member of the binary, at a distance of about $r_{t}$ from the BH, results in a change in gravitational energy of $G M a / r_{t}^{2} \sim v_{0}^{2}(M / m)^{1 / 3}$; this is comparable to the variation in the kinetic energy. As we have done, it should be easier to discuss the overall effect in the energy domain. In our formula, the energy change (17) includes both the variation of kinetic energy and potential energy. For prograde orbits, the kinetic and potential terms cooperate and the net energy change is larger, the member on the "outside track" is expected to be ejected and its partner is captured (the "outside track" could be well defined, especially when the orbital energy is large because the duration of the encounter is much shorter than the binary rotation timescale). On the other hand, for retrograde orbits the variation in the gravitational energy would counteract that in the kinetic energy.

\section{NUMERICAL RESULTS}

In this paper, we focus on results for circular coplanar binaries, though our formulae can be used to study the evolution of a binary with arbitrary orbital parameters. The orbit of a binary is assumed to be initially circular in the comoving frame of the binary center of mass. The center of mass of the binary is in a prograde or retrograde orbit around the BH (see SKR for the details of the numerical setup).

For $M / m \gg 1$, the problem can be reduced to the motion of a single particle in a time-dependent potential ("the restricted three-body approximation") described by Equations (5)-(7) and (10). The energy change, Equation (19), depends only on the penetration factor $D$, the eccentricity $e$, the initial binary phase $\phi$, and the binary rotation direction. As we have shown, when a binary member is captured by the $\mathrm{BH}$ and the other is ejected, we can further reduce the number of the parameters by assuming $e=1$ to approximate the additional energy. The effect of the eccentricity $e \neq 1$ is taken into account through the non-zero initial orbital energy of the center of mass. This method (22) will be called "the parabolic approximation." For a large orbital energy $\bar{E} \gg(M / m)^{1 / 3}\left(m / m_{1}\right)\left(m / m_{2}\right)$, "the sudden disruption approximation" (24) would become valid, but the three-body exchange reaction does not take place in this regime. In all the numerical codes, the time evolution of objects are evaluated by using a fourth-order Runge-Kutta integration scheme.

\subsection{Energy Change and Probability Distributions}

We have tested the restricted three-body approximation against the full three-body simulations of a binary evolving around a massive object (SKR). The full three-body orbit is accurately reproduced by the approximation equations, and the energy change, for example, differs at a $0.1 \%$ level when the exchange reaction happens. The comparison of the energy change between the full three-body and restricted three-body results is shown in Figure 1. Since they are in excellent agreement, in the following discussion, we will use the restricted three-body approximation to test the parabolic and the sudden disruption approximation.
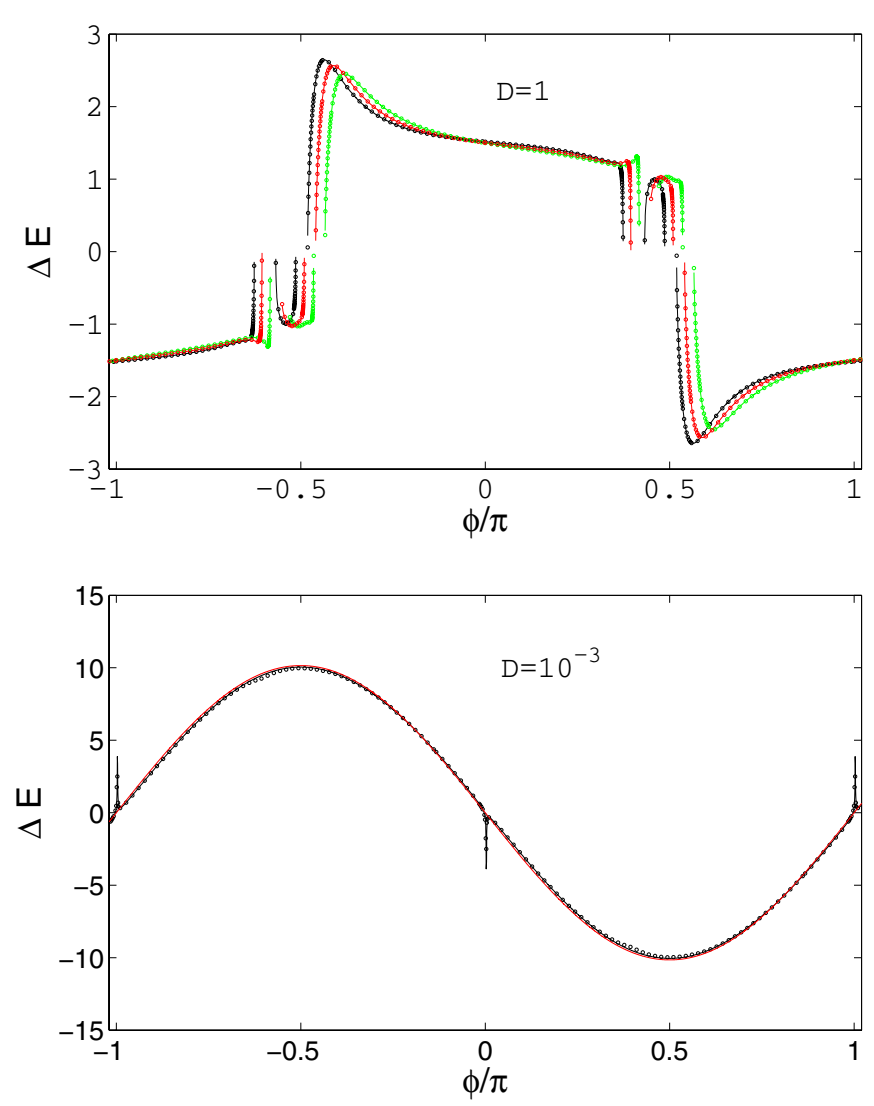

Figure 1. Energy change as a function of $\phi$. Top panel $(D=1)$ : the restricted three-body approximation for $e=0.9$ (green solid line), 1 (red solid line), and 1.1 (black solid line). The full three-body calculations (circles). Bottom panel ( $D=10^{-3}$ and $e=1.1$ ): the restricted three-body approximation (black solid line), the full three-body calculations (black circles), and the sudden disruption approximation (red solid line). In the full three-body calculations, $M / m=10^{6}$ and $m_{1} / m_{2}=3$ are assumed and the energy change is evaluated as $\Delta E=\left(m_{1} / m\right) E_{2}-\left(m_{2} / m\right) E_{1}$. Prograde orbits are assumed for all of the calculations. Energy is in units of $\left(G m_{1} m_{2} / a\right)(M / m)^{1 / 3}$.

(A color version of this figure is available in the online journal.)

In Figure 1, one could notice the symmetry $\Delta \bar{E}(\phi+\pi)=$ $-\Delta \bar{E}(\phi)$. The top panel shows that the energy change for $|e-1| / D=0.1$ is very similar to the parabolic case with the same $D$, especially if we take into account the phase shifts. ${ }^{8}$ When the three-body exchange reaction happens, the value $|e-1| / D \lesssim(m / M)^{1 / 3}\left(m_{\mathrm{par}} / m\right) \ll 1$ should be very small, in such a case non-parabolic results tend toward a perfect overlapping with the parabolic results. In the bottom panel, the energy change in the high-energy regime, $(e-1) / D=100$, is shown. The sudden disruption approximation well reproduces the three-body results, except for some notable spikes.

Most binaries are disrupted at the tidal encounter with a $\mathrm{BH}$. However, there are always finite-phase regions where binaries

8 The initial distance of the binary center of mass to the $\mathrm{BH}$ is assumed to be $r_{0}=15 r_{t}$ for the parabolic calculations in the top panel of Figure 1 . As long as a simulation starts at a large enough radius $r_{0} \gg r_{t}$, the results are largely independent of it. However, a problem arises when we compare the phase dependence. If we assume the same initial radius for the non-parabolic cases, it takes a slightly different time for the binary to reach the vicinity of the $\mathrm{BH}$, the binary interacts with the $\mathrm{BH}$ with a slightly different binary phase. We have adjusted the initial radii as the periapsis passage happens at the same time $\left(r_{0} \sim 12.2 r_{t}\right.$ for $e=0.9$ and $\sim 17.4 r_{t}$ for $e=1.1$ ). Since this adjustment has been done neglecting the $\mathrm{BH}$ tidal field, the actual phase at the periapsis is still different from the parabolic case. This induces the phase shifts in the figure. In the bottom panel of Figure 1, $r_{0}=15 r_{t}$ is assumed for the restricted three-body calculations, and in each case the binary phase is adjusted as the results take the maximum value at $\phi=-\pi / 2$. 

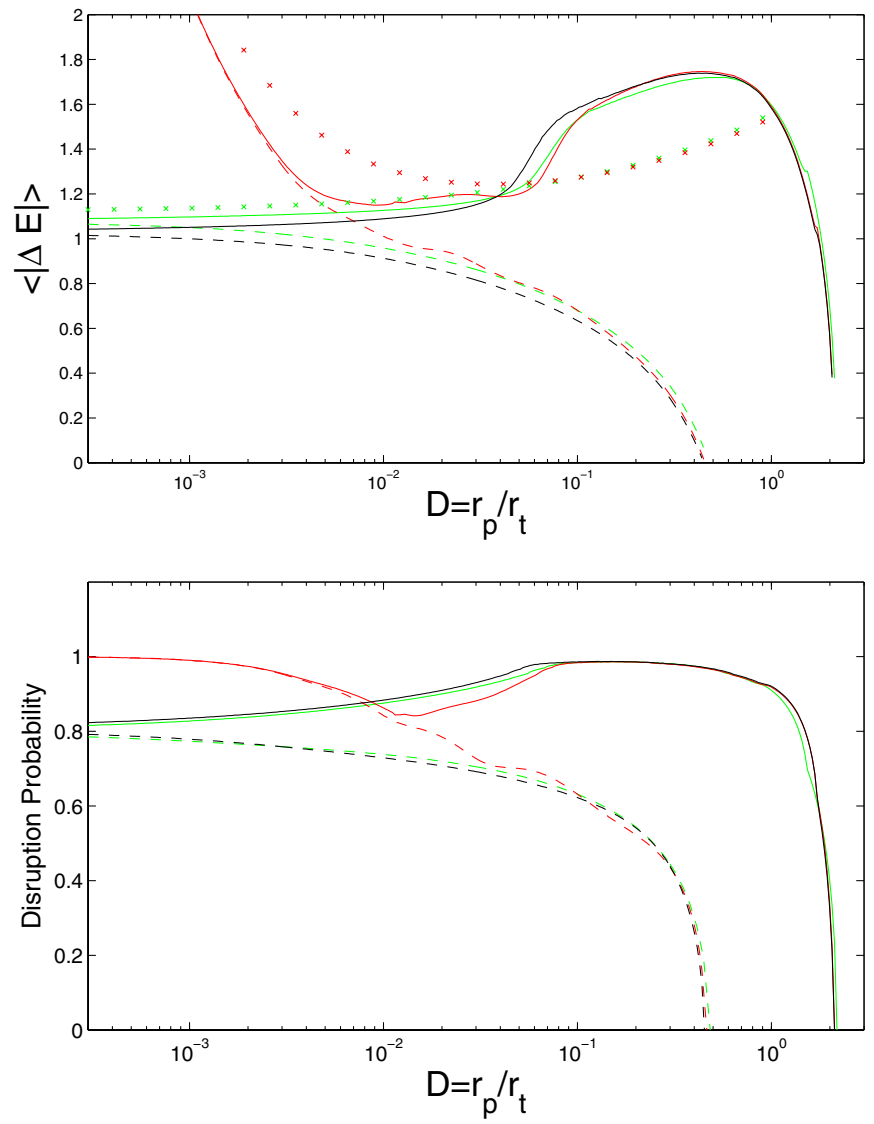

Figure 2. Phased-averaged energy change (top panel) and disruption probability (bottom panel) as a function of $D$. Results for the restricted three-body approximation are shown for $e=1$ (black lines), $e=1.01$ (red lines), and $(e-1) / D=0.1$ (green lines). Prograde (solid lines) and retrograde (dashed lines). The sudden disruption approximation is shown for prograde orbits with $e=1.01$ (red crosses) and $(e-1) / D=0.1$ (green crosses). Energy is in units of $\left(G m_{1} m_{2} / a\right)(M / m)^{1 / 3}$.

(A color version of this figure is available in the online journal.)

survive (SKR). The narrow gaps in the top panel of Figure 1 correspond to such regions. Although it is not evident in the bottom panel, very narrow gaps exist just in the middle of the spikes. The high-energy regime is usually realized with a deep penetrator $D \ll 1$. In such a case, we can ignore the self-gravity term $\mathbf{r} / r^{3}$ in Equations (5)-(7) and free solutions are obtained. Actually, one of the free solutions, $(x, y) \propto(-\sin f, e+\cos f)$, which corresponds to the case that binary members have the same trajectory but are slightly separated in time, dominates around the periapsis passage (SKR). Then, the binary is once disrupted at the tidal encounter, but after the periapsis passage at $t=0$, they come close to each other. If we fine tune the initial binary phase, they form a binary again. This produces the narrow gaps at the spikes. If the binary phase is slightly different from the fine-tuned values, they almost form a binary, but they eventually break up. The additional work at $t>0$ due to the mutual forces between the binary members produces the notable spikes.

The top panel of Figure 2 shows the phase-averaged absolute value of the energy change $\langle|\Delta \bar{E}|\rangle$ as a function of the penetration factor $D$. The average is taken over the phase space where binaries are disrupted. We alternatively fix the orbital eccentricity or the orbital energy $\bar{E} \propto(e-1) / D$. For a given eccentricity, a smaller $D$ corresponds to a larger orbital energy, while for a given orbital energy, it corresponds to $e \rightarrow 1$. For

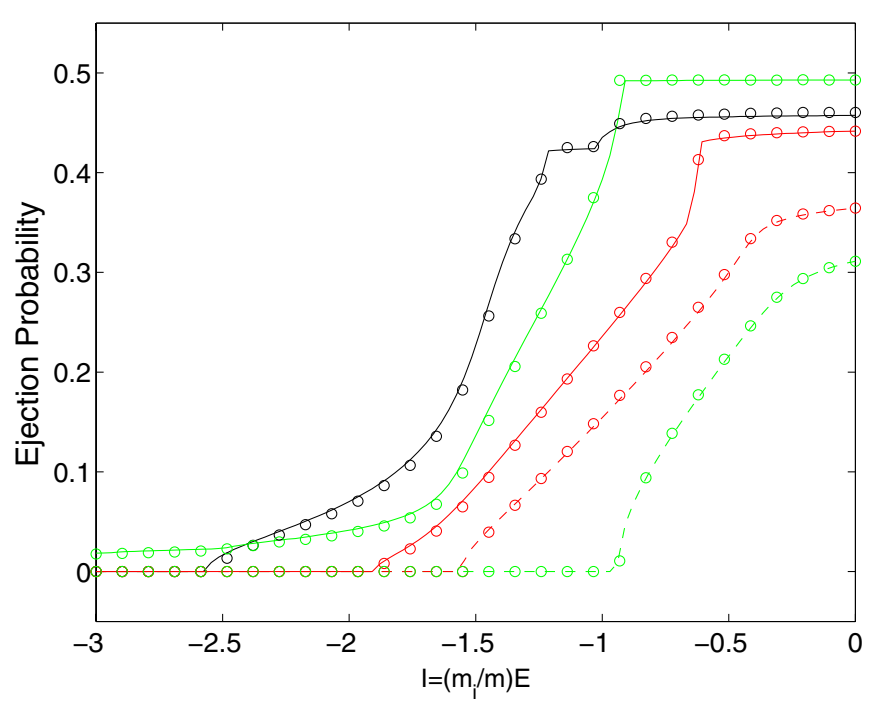

Figure 3. Ejection probability as a function of $\bar{I}=\left(m_{i} / m\right) \bar{E}$. The parabolic approximation is shown for $D=1$ (black line), $10^{-1}$ (green lines), and $10^{-2}$ (red lines). The solid and dashed lines indicate prograde orbit and retrograde orbit results, respectively. The circles show the restricted three-body results for the corresponding cases with $M / m=10^{6}$ and $m_{i} / m=1 / 4$. Energy is in units of $\left(G m_{1} m_{2} / a\right)(M / m)^{1 / 3}$.

(A color version of this figure is available in the online journal.)

$(e-1) / D \lesssim 1$, the energy change remains of the order of unity as we expect from Equation (23) (see the black and green lines and the green crosses for the whole disruption range, and the red lines and red crosses for $\left.D \gtrsim 10^{-2}\right)$. For $(e-1) / D \gtrsim 1$, instead, $\langle|\Delta \bar{E}|\rangle$ increases toward smaller $D$ (the red lines and red crosses for $\left.D \lesssim 10^{-2}\right)$. Correspondingly, the disruption probability (bottom panel) becomes almost $100 \%$ for $(e-1) / D \gtrsim 1$. On the other hand, for the fixed energies $(e-1) / D=0$ and 0.1 , the disruption chance is about $80 \%$ even in the deep penetration limit $D \ll 1$ (the black and green lines). Note that in the sudden disruption approximation the disruption probability is $100 \%$ by definition, and we do not show it in the bottom panel. For our choice of $(e-1) / D=0.1$, the behavior of $\langle|\Delta \bar{E}|\rangle$ always remains very similar to the parabolic orbit case, even when we use the sudden disruption approximation especially for small $D$ (the green crosses in the top panel). The same applies to the disruption probability function, but the sudden disruption approximation overestimates the disruption probability. As it is well known, the retrograde binaries tend to be more stable against the tidal encounter.

For a given initial energy of a binary member $\bar{I}=\left(m_{i} / m\right) \bar{E}$ (in the following discussion we drop the subscript $i$ of the initial energy for simplicity), considering the symmetry of $\Delta \bar{E}(\phi)$, the probability $P_{\text {eje }}(\bar{I})$ that the member is ejected after the tidal encounter is determined by the fraction of the binary phase region $[0,2 \pi]$ that satisfies $\Delta \bar{E}(\phi)<\bar{I}$, while the capture probability $P_{\text {cap }}(\bar{I})$ is determined by the fraction satisfying $\Delta \bar{E}(\phi)>\bar{I}$. The sum is equal to the disruption probability: $P_{\text {dis }}=P_{\text {eje }}+P_{\text {cap }}$. Since $P_{\text {eje }}(-\bar{I})=P_{\text {cap }}(\bar{I})$, once we evaluate the ejection probability $P_{\text {eje }}(\bar{I})$ for negative and zero energy, the distribution for positive energy $P_{\text {eje }}(\bar{I})=2 P_{\text {eje }}(0)-P_{\text {eje }}(-\bar{I})$ and the capture probability for any energy $P_{\text {cap }}(\bar{I})=P_{\text {eje }}(-\bar{I})$ are also obtained. The disruption probability is $P_{\text {dis }}=2 P_{\text {eje }}(0)$.

We show in Figure 3 how the ejection probability of a binary member behaves for different penetration factors and binary orientations. Since the orbital energy and the eccentricity are not 

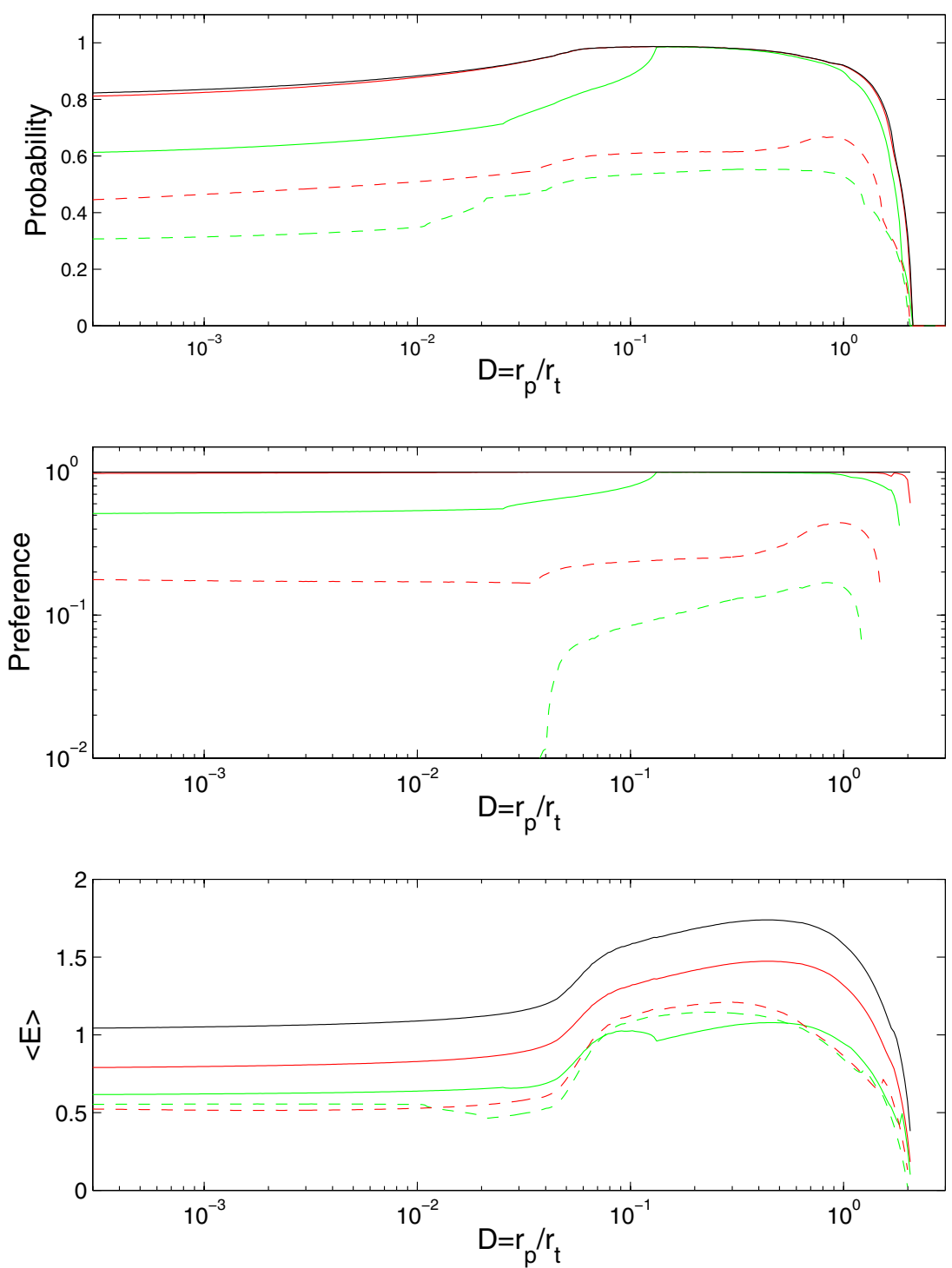

Figure 4. Ejection probability, preference, and energy. Parabolic orbit (black solid), $\left|r_{a} / r_{t}\right|=500$ (red solid), 200 (green solid), 130 (red dashed), and 100 (green dashed). The parabolic approximation is used. The ejection preference is the ratio of the ejection probabilities (the primary star/the secondary star). The ejection energy is evaluated by taking the phase average of all the ejected cases. Energy is in units of $\left(G m_{1} m_{2} / a\right)(M / m)^{1 / 3}$.

(A color version of this figure is available in the online journal.)

independent parameters when $D$ is fixed, $e-1 \propto \bar{E}$, we need to assume a smaller eccentricity, in principle, for a larger negative orbital energy $\bar{I}$ (or larger negative $\bar{E}$ ) to estimate the energy change $\Delta \bar{E}(\phi)$, the disruption probability, and the ejection probability. However, the energy change and the disruption probability are not so sensitive to the eccentricity, we thus evaluate them by using parabolic orbit results (the parabolic approximation) when the three-body exchange reaction happens. In the figure, the parabolic approximation calculations (the lines) are in a good agreement with the restricted three-body calculations (the circles). For prograde orbits, the energy change $\Delta \bar{E}(\phi)$ is more sensitive to the phase $\phi$ around zero points, only very narrow phase regions satisfy $|\Delta \bar{E}| \lesssim 1$ (see Figure 1, top panel), then the ejection probabilities have a plateau around $\bar{I}=0$. The long low energy tail of the prograde $D=0.1$ case (the green solid line) reflects the fact that the energy gain would be quite large for a narrow binary phase region for this case. The ejection (capture) probability for $-\infty<\bar{I}<\infty$ is a monotonically increasing (decreasing) function of the energy $\bar{I}$. The probabilities rapidly change around $|\bar{I}| \sim 1$ and their function form depends mainly on $D$ and the binary rotation direction.

\subsection{Probability Distributions for Various Semimajor Axes}

When we discuss an actual astrophysical system, it is more physically intuitive to use the semimajor axis rather than the energy $\bar{I}$ or $\bar{E}$. By specifying the semimajor axis and the mass ratios, the ejection (or capture) probability and the energy after the disruption can be evaluated. The orbital energy $\bar{E}$ is given by

$$
\bar{E}=-2.7\left(\frac{r_{a} / r_{t}}{100}\right)^{-1}\left(\frac{M / m}{10^{6}}\right)^{1 / 3}\left(\frac{m_{1} / m}{3 / 4}\right)^{-1}\left(\frac{m_{2} / m}{1 / 4}\right)^{-1}
$$

where $M / m=10^{6}$ and $m_{1} / m_{2}=3$ will be assumed in this section.

Figure 4 shows the characteristics of the ejection process when a binary approaches the $\mathrm{BH}$ in elliptic orbits. To evaluate 
the ejection probability (the top panel), we have not distinguished which member is ejected. Since both members are never ejected together when $\bar{E}<0$, it is just the sum of the ejection probabilities of the two members. For parabolic orbits, one of the members is always ejected if the binary is disrupted, then the black solid line indicates the disruption probability. As $r_{a} / r_{t}$ becomes smaller, the deviation of the orbit from the parabolic one becomes larger. The effect of the nonzero orbital energy is expected to become significant around $r_{a} / r_{t} \sim(M / m)^{1 / 3}\left(m / m_{2}\right) \sim 400$, and the ejection probability rapidly decreases. The middle panel indicates which member in a binary is ejected more frequently. There is no preference in the parabolic case. However, the secondary is preferentially ejected for small $r_{a} / r_{t}$. The phase-averaged ejection energy is shown in the bottom panel. For parabolic orbits this is equivalent to the phase-averaged energy change $\langle|\Delta \bar{E}|\rangle$.

If we consider $P_{\text {cap }}(\bar{I})=P_{\text {eje }}(-\bar{I})$, it is possible to interpret Figure 4 as the capture probability and capture preference for the hyperbolic orbit cases with the same semimajor axes in absolute value. The phase-averaged energy (the bottom panel) gives the absolute values of the averaged capture energies.

\section{IRREGULAR SATELLITES AROUND GIANT PLANETS}

Over 150 satellites are orbiting the giant planets in the solar system. About one-third of these are classified as regular, with nearly circular and planar orbits. The majority of the satellites, however, are irregular ones which are more distant from their planet and typically have larger eccentricities and/or inclination. Interestingly, a large fraction of the irregular satellites orbit their planet in the retrograde direction (Jewitt \& Haghighipour 2007). Triton, Neptune's largest moon, is among them. Because of the retrograde orbit and composition similar to Pluto's, Triton is thought to have been captured from the Kuiper belt. Recently, Agnor \& Hamilton (2006) demonstrated that the gravitational encounter between Neptune and a binary system, which had included Triton as a member, is an effective mechanism to capture Triton. Since the mass ratio between Neptune $m_{N} \sim 17.2 M_{\oplus}$ and Triton $m_{T} \sim 3.58 \times 10^{-3} M_{\oplus}$ is reasonably large $m_{N} / m_{T} \sim 4800$, we here revisit the capture process from the point of view of the restricted three-body problem.

We consider an encounter between Neptune and a binary system with $m_{1}=m_{T}, m_{2}=0.1 m_{T}$, and $a=20 R_{T}$. The binary is assumed to be in a prograde hyperbolic orbit with the periapsis $r_{\mathrm{p}}=8 R_{N}$ where $R_{T} \sim 1.35 \times 10^{3} \mathrm{~km}$ and $R_{N} \sim 2.46 \times 10^{4} \mathrm{~km}$ are the radii of Triton and Neptune, respectively. This set of the parameters is identical to what Agnor \& Hamilton (2006) have assumed to obtain their Figure 2. Since the tidal radius is about $r_{t} \sim 18 R_{N}$, the penetration factor for the orbit is $D \sim 0.45$. For a given encounter velocity at infinity $v_{\infty}$, we evaluate the binary disruption chance and the capture probability of the binary members. The velocity at infinity $v_{\infty}=1 \mathrm{~km} \mathrm{~s}^{-1}$ is related to the semimajor axis of the hyperbolic orbit as

$$
\begin{aligned}
\left|r_{a} / r_{t}\right|= & 16\left(\frac{v_{\infty}}{1 \mathrm{~km} \mathrm{~s}^{-1}}\right)^{-2}\left(\frac{a}{20 R_{T}}\right)^{-1}\left(\frac{m}{1.1 m_{T}}\right) \\
& \times\left(\frac{M / m}{4.37 \times 10^{3}}\right)^{2 / 3},
\end{aligned}
$$

which is about the critical value $\left|r_{a} / r_{t}\right| \gtrsim(M / m)^{1 / 3} \sim 16$ for the three-body exchange reaction. The numerical results are
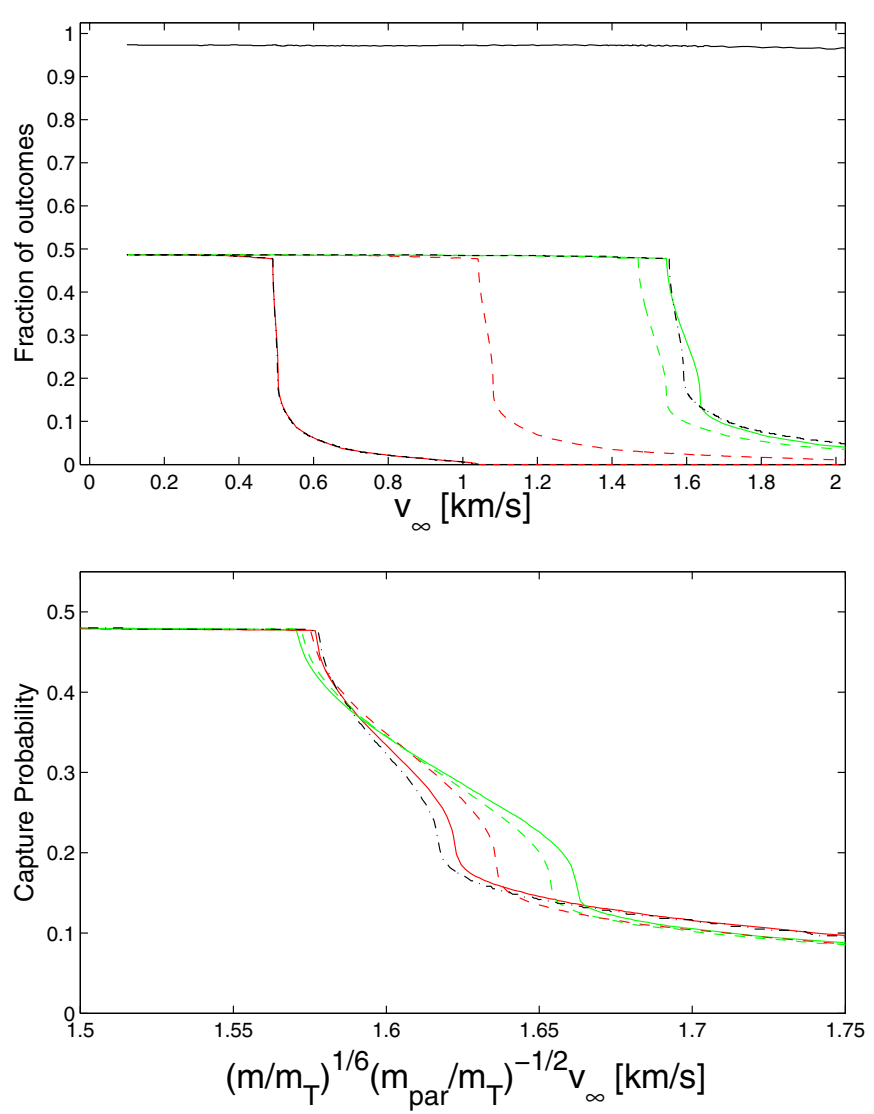

Figure 5. Top panel: the restricted three-body approximation: binary disruption chance (black solid), capture chance for $m_{2}=0.1 m_{T}$ : the primary (red solid) and the secondary (green solid), capture chance for $m_{2}=m_{T} / 2$ : the primary (red dashed) and the secondary (green dashed). The parabolic approximation for $m_{2}=0.1 m_{T}$ (black dash-dotted). Bottom panel: capture chance as a function of the scaled velocity. $D \sim 0.45$ is assumed.

(A color version of this figure is available in the online journal.)

shown in Figure 5, and the relevant case (the solid lines in the top panel) is in good agreement with Figure 2 in Agnor \& Hamilton (2006). Since the disruption chance depends mainly on $D$, for the fixed $D$, it is constant $\sim 97 \%$. For high velocities, the probability for the primary drops sharply, while it is still $\sim 50 \%$ for the secondary because of its smaller initial energy $\bar{I}$. If we assume a larger secondary mass $m_{2}=m_{T} / 2$, then the energy change $\Delta E$ at the encounter would be larger. The $50 \%$ capture probability of the primary now extends to higher velocities, while the capture rate of the secondary is similar because the primary mass is fixed (the dashed lines). The black dash-dotted lines indicate the capture probabilities obtained by using the parabolic approximation for $m_{2}=0.1 m_{T}$. Even at the high velocity $v_{\infty} \sim 1.6 \mathrm{~km} \mathrm{~s}^{-1}$ or equivalently high eccentricity $e-1=D(m / M)^{2 / 3}\left(a v_{\infty}^{2} / G m\right) \sim 7 \times 10^{-2}$, it reasonably agrees with the restricted three-body results. In the bottom panel, the capture probability is shown as a function of a scaled velocity $\left(m / m_{T}\right)^{1 / 6}\left(m_{\text {par }} / m_{T}\right)^{-1 / 2} v_{\infty}=$ $\left(m_{N} / m_{T}\right)^{1 / 6}\left(2 \bar{I} G m_{T} / a\right)^{1 / 2}$. Although the difference among them is small, if the parabolic approximation is used for all the cases, then the solid and dashed lines should perfectly overlap with the black dash-dotted line.

At the disruption, each member is captured with $50 \%$ probability if the initial energy $\bar{I}$ is close to zero. Using the relation $P_{\text {cap }}(\bar{I})=P_{\text {eje }}(-\bar{I})$, Figure 3 also shows that at high energies the capture is very rare. A transition occurs at an intermediate 
energy as shown in Figure 3. For $D=0.45$ prograde orbits, the capture probability sharply drops from $\sim 50 \%$ around $\bar{I} \sim 1.4$. The critical value of $v_{\infty}$ at which the capture probability of a binary member $m_{1}$ drops from $\sim 50 \%$ is given by

$$
\begin{aligned}
v_{\infty, \text { crit }}= & \sqrt{\frac{2 \bar{I} G m_{2}}{a}}\left(\frac{M}{m}\right)^{1 / 6} \sim 0.49\left(\frac{\bar{I}}{1.4}\right)^{1 / 2}\left(\frac{m_{2}}{0.1 m_{T}}\right)^{1 / 2} \\
& \times\left(\frac{a}{20 R_{T}}\right)^{-1 / 2}\left(\frac{M / m}{4.37 \times 10^{3}}\right)^{1 / 6} \mathrm{~km} \mathrm{~s}^{-1} .
\end{aligned}
$$

This estimate well explains the critical velocities in Figure 5. If Triton has a heavier companion and/or a member of a harder binary, then the critical velocity could be higher, provided the binary is disrupted by Neptune: $D \lesssim 1$. The semimajor axis of the captured member $m_{1}$ is

$$
\begin{aligned}
r_{a}= & \frac{a}{2\left|\bar{E}_{1}\right|}\left(\frac{M}{m_{2}}\right)\left(\frac{m}{M}\right)^{1 / 3} \sim 1.6 \times 10^{3} R_{N}\left|\bar{E}_{1}\right|^{-1} \\
& \times\left(\frac{a}{20 R_{T}}\right)\left(\frac{M / m_{2}}{4.80 \times 10^{4}}\right)\left(\frac{M / m}{4.37 \times 10^{3}}\right)^{-1 / 3}
\end{aligned}
$$

where $\left|\bar{E}_{1}\right|$ is about $|\Delta \bar{E}| \sim 1$. After capture, the orbit of Triton needs to shrink to the current observed $r_{a} \sim 14 R_{N}$ and $e \sim 10^{-5}$, either through tides or other means (Correia 2009; Nogueira et al. 2011). The critical velocity and the semimajor axis for the secondary $m_{2}$ are obtained by exchanging the subscript 1 and 2 in Equations (27) and (28).

Although a detailed modeling of the solar system is beyond the scope of this paper, the Sun cannot be generally ignored in the capture process (e.g., Philpott et al. 2010; Gaspar et al. 2011). Since the tidal radius $r_{t} \sim 18 R_{N}\left(\mathrm{~m} / \mathrm{m}_{T}\right)^{-1}\left(a / 20 R_{T}\right)$ is much smaller than the radius of Neptune's Hill sphere $r_{H} \sim 4700 R_{N}$, Neptune completely dominates the attraction of a binary during the tidal encounter. Tidal effects from the Sun are negligible for the disruption process itself, which is the main focus of the paper. However, the permanent capture of Triton requires an additional condition that after the tidal breakup a capture orbit should be well inside the Hill sphere. Detailed numerical simulations show that retrograde satellites (those orbiting in the opposite sense as Neptune orbits the Sun $^{9}$ ) of Neptune are more stable than prograde ones, and they are stable to distance of $r_{\text {stable }} \sim 0.4 r_{H}$ (Nesvorný et al. 2003; Holman et al. 2004). Capture orbits are more eccentric than assumed in the numerical simulations, the stability region might be slightly smaller. Considering that the apocenter distance is about twice the semimajor axis (28) for highly eccentric orbits, the binary which had included Triton as a member should satisfy a relation

$$
\left(\frac{a}{20 r_{T}}\right)\left(\frac{m_{2}}{0.1 m_{T}}\right)^{-1}\left(\frac{m}{1.1 m_{T}}\right)^{1 / 3} \lesssim 0.4\left(\frac{r_{\text {stable }}}{0.3 r_{H}}\right),
$$

where we have used $|\Delta \bar{E}| \sim 1$. A smaller value of the ratio $a / m_{2}$ gives a smaller apocenter distance and a higher critical velocity (27). As shown in the top panel of Figure 2 (the black solid

\footnotetext{
9 In the three-body encounter discussion, prograde motion means that the binary center of mass is orbiting around a massive object in the same sense as the binary members rotate around their center of mass. Then, a moon can be captured in a retrograde orbit around Neptune (the orbit is in the direction opposite to the rotation of Neptune) after the tidal breakup of a prograde binary (the angular momentum of the binary around Neptune and of a binary member around the binary center of mass are aligned).
}

and dashed lines), the phase-averaged value $\langle|\Delta \bar{E}|\rangle$ is slightly larger than unity for prograde binaries, and slightly smaller for retrograde binaries when the disruption probability is high. For a given encounter velocity, a retrograde orbit of the binary center of mass around Neptune with a prograde binary rotation is optimal to produce a stable capture orbit.

The ellipticity $\epsilon_{N} \sim 1.7 \times 10^{-2}$ of Neptune induces a small deviation in its own gravitational potential from the point-mass estimate, especially in a non-planar configuration. However, the deviation in the attraction force $\Delta F / F$ and the tidal acceleration $\Delta a_{t} / a_{t}$ is of the order of $\left(R_{N} / r_{t}\right)^{2} \epsilon_{N} \sim 5 \times 10^{-5}$ at the tidal radius. The effect is clearly negligible.

\section{CONCLUSIONS}

We have discussed how the members of a binary are ejected or captured after a tidal encounter with a massive object. We have shown that the ejection and capture dynamics can be well understood in the framework of the restricted three-body approximation. When the three-body exchange reaction happens, the orbit of the center of mass of the binary should be almost parabolic $|1-e| \lesssim D(m / M)^{1 / 3}\left(m_{\text {par }} / m\right) \ll 1$ or equivalently the semimajor axis is large $\left|r_{a} / r_{t}\right| \gtrsim(M / m)^{1 / 3}\left(m / m_{\text {par }}\right)$. The essential quantity to characterize the disruption process is the energy change $\Delta \bar{E}$ at the encounter, which practically depends only on three parameters: the penetration factor $D=r_{\mathrm{p}} / r_{t}$, the binary rotation direction, and the binary phase $\phi$. Except for the phase shift of $\pi$, the energy change is exactly the same for the two members with arbitrary mass ratio.

In principle, for any positive (negative) orbital energy $E$ of the center of mass of a binary, the heavier member has more chance to be ejected (captured), because it carries a larger fraction of the orbital energy. However, if the orbital energy is close to zero, the difference between their ejection (capture) probabilities becomes small, and there is practically no ejection and capture preference. For a parabolic orbit, each member is ejected in exactly $50 \%$ of the cases. The preference becomes significant when the absolute value of the energy $|E|$ is comparable to the typical energy change $\left(G m_{1} m_{2} / a\right)(M / m)^{1 / 3}$. On the other hand, if $|E|$ is much larger than the typical energy change, they are both ejected for $E>0$ or captured for $E<0$, and there is no ejection or capture preference.

Corresponding to the typical energy change, we can define a critical encounter velocity $v_{\infty, \text { crit }}=\left(2 \bar{I} G m_{\text {par }} / a\right)^{1 / 2}(M / m)^{1 / 6}$ for the capture process where the critical initial energy $\bar{I} \sim$ 0.5-1.5 mainly depends on the penetration factor and the orientation of the binary. Since the distribution of the energy change is the same for the two members, the secondary star has a higher critical velocity. The tidal encounter might be responsible for the capture of not only Triton but also other irregular satellites in the solar system (Morbidelli 2006, but see also Vokrouhlický et al. 2008; Philpott et al. 2010. The stability of capture orbits for the solar perturbations also should be taken into account). However, the other irregular satellites are much lighter than Triton. The capture mechanism $v_{\infty, \text { crit }} \propto m_{\text {par }}^{1 / 2}$ requires that these irregular satellites have been in a binary with a very massive partner, and that the less massive member has been predominantly captured.

If a binary has a large orbital energy $E \gg(M / m)^{2 / 3}\left(G m^{2} / a\right)$, then the disruption would happen slightly inside the usual tidal radius at which the $\mathrm{BH}$ tidal forces become comparable to the binary mutual gravity forces. The abrupt disruption approximation provides a good estimate of the energy change, 
and the typical energy change in the high-energy regime is much larger than in the low-energy regime. However, the change is not significant compared to the initial energy $E$, and the tidal encounter is not an efficient acceleration process anymore. In the high-energy regime, both members are ejected after the $\mathrm{BH}$ encounter.

In recent years, observations have identified a remarkable number of hypervelocity stars (Brown et al. 2009; Tillich et al. 2011). The distribution of line-of-sight velocities of hypervelocity star candidates shows a long tail in the highvelocity region $\left(v>275 \mathrm{~km} \mathrm{~s}^{-1}\right)$ which includes comparable numbers of unbound and bound stars assuming that the escape velocity of the Galaxy at $50 \mathrm{kpc}$ is $\sim 360 \mathrm{~km} \mathrm{~s}^{-1}$ (Brown et al. 2009; Kenyon et al. 2008). Since the initial orbital energy is negligible when the three-body exchange reaction happens, the velocity distribution should reflect the distribution of the energy change $\Delta E$ at the tidal encounter (and the Galactic potential) with its dependence on the periapsis distance, the binary orientation, and phase.

Binaries are supplied to the $\mathrm{BH}$ at the Galactic center predominantly from its radius of influence $r_{h} \sim$ a few pc or even beyond it (e.g., Perets et al. 2007; another interesting possibility is that they might arise from the nuclear stellar disk, e.g., Madigan et al. 2011). Those that come from about the radius of influence are on elliptical orbits. Since the radius of influence is much larger than the tidal radius, the preferential ejection for high- and low-mass members $\left(m_{1}>m_{2}\right)$ is therefore irrelevant for stellar binaries $\left(m_{1} / m_{2} \lesssim 10\right)$ if the binary semimajor axis is smaller than $\sim 1$ AU. However, stars with planets $\left(m_{1} / m_{2} \gtrsim 10^{3}\right)$ of a semimajor axis of $1 \mathrm{AU}$ will predominantly eject the planets.

We thank the referee, Hagai Perets, for his constructive comments, and Mostafa Ahmadi, Phil Armitage, Witold Maciejewski, and Ruth Murray Clay for useful discussion. This research was supported by STFC, ERC, and IRG grants, and Packard, Guggenheim, and Radcliffe fellowships.

\section{REFERENCES}

Agnor, C. B., \& Hamilton, D. P. 2006, Nature, 441, 192

Antonini, F., Lombardi, J. C., Jr., \& Merritt, D. 2011, ApJ, 731, 128

Ayal, S., Livio, M., \& Piran, T. 2000, ApJ, 545, 772
Bloom, J. S., Giannios, D., Metzger, B. D., et al. 2011, Science, 333, 203

Bromley, B. C., Kenyon, S. J., Geller, M. J., et al. 2006, ApJ, 653, 1194

Brown, W., Geller, M., \& Kenyon, S. 2009, ApJ, 690, 1639

Brown, W. R., Geller, M. J., Kenyon, S. J., \& Kurtz, M. J. 2005, ApJ, 622, L33

Burrows, D. N., Kennea, J. A., Ghisellini, G., et al. 2011, Nature, 476, 421

Correia, A. C. M. 2009, ApJ, 704, L1

Donley, J. L., Brandt, W. N., Eracleous, M., \& Boller, Th. 2002, AJ, 124, 1308

Edelmann, H., Napiwotzki, R., Heber, U., Christlieb, N., \& Reimers, D. 2005, ApJ, 634, L181

Evans, C., \& Kochanek, C. S. 1989, ApJ, 346, L13

Gaspar, H. S., Winter, O. C., \& Vieira Neto, E. 2011, MNRAS, 415, 1999

Genzel, R., Eisenhauer, F., \& Gillessen, S. 2010, Rev. Mod. Phys., 82, 3121

Ghez, A. M., Salim, S., Hornstein, S. D., et al. 2005, ApJ, 620, 744

Ginsburg, I., \& Loeb, A. 2006, MNRAS, 368, 221

Gould, A., \& Quillen, A. C. 2003, ApJ, 592, 935

Grupe, D., Beuermann, K., Mannheim, K., et al. 1995, A\&A, 299, L5

Gualandris, A., Portegies Zwart, S., \& Sipior, M. S. 2005, MNRAS, 363, 223

Guillochon, J., Ramirez-Ruiz, E., Rosswog, S., \& Kasen, D. 2009, ApJ, 705, 844

Heggie, D. C. 1975, MNRAS, 173, 729

Hills, J. G. 1975, AJ, 80, 809

Hills, J. G. 1988, Nature, 331, 687

Hirsch, H. A., Heber, U., O’Toole, S. J., \& Bresolin, F. 2005, A\&A, 444, L61

Holman, M. J., Kavelaars, J. J., Grav, T., et al. 2004, Nature, 430, 865

Jewitt, D., \& Haghighipour, N. 2007, ARA\&A, 45, 261

Kenyon, S. J., Bromley, B. C., Geller, M. J., \& Brown, W. R. 2008, ApJ, 680, 312

Kobayashi, S., Laguna, P., Phinney, E. S., \& Mészáros, P. 2004, ApJ, 615, 855

Komossa, S., \& Bade, N. 1999, A\&A, 343, 775

Laguna, P., Miller, W. A., Zurek, W. H., \& Davies, M. B. 1993, ApJ, 410, L83

Landau, L. D., \& Lifshitz, E. M. 1976, Mechanics (Oxford: ButterworthHeinemann)

Levan, A. J., Tanvir, N. R., Cenko, S. B., et al. 2011, Science, 333, 199

Lodato, G., \& Rossi, E. M. 2011, MNRAS, 410, 359

Madigan, A. M., Hopman, C., \& Levin, Y. 2011, ApJ, 738, 99

Morbidelli, A. 2006, Nature, 441, 162

Nesvorný, D., Alvarellos, J. L. A., Dones, L., \& Levison, H. F. 2003, AJ, 126, 398

Nogueira, E., Brasser, R., \& Gomes, R. 2011, Icarus, 214, 113

Perets, H. B., Hopman, C., \& Alexander, T. 2007, ApJ, 656, 709

Philpott, C. M., Hamilton, D. P., \& Agnor, C. B. 2010, Icarus, 208, 824

Rees, M. 1988, Nature, 333, 523

Sari, R., Kobayashi, S., \& Rossi, E. M. 2010, ApJ, 708, 605 (SKR)

Sesana, A., Haardt, F., \& Madau, P. 2007, MNRAS, 379, L45

Strubbe, L. E., \& Quataert, E. 2009, MNRAS, 400, 2070

Tillich, A., Heber, U., Geier, S., et al. 2011, A\&A, 527, A137

Tutukov, A. V., \& Fedorova, A. V. 2009, Astron. Rep, 53, 839

Vokrouhlický, D., Nesvorný, D., \& Levison, H. F. 2008, AJ, 136, 1463

Yu, Q., \& Tremaine, S. 2003, ApJ, 599, 1129

Zauderer, B. A., Berger, E., Soderberg, A. M., et al. 2011, Nature, 476, 425

Zhang, F., Lu, Y., \& Yu, Q. 2010, ApJ, 722, 1744 